CHRISTIAN CHÁVEZ LÓPEZ

FACULTAD DE ARTES Y DISEÑO

UNIVERSIDAD NACIONAL AUTÓNOMA DE MÉXICO,

CIUDAD DE MÉXICO, MÉXICO.

CCHAVEZL@FAD.UNAM.MX

Nota de la autora:

Este artículo es parte de los avances de mi investigación de tesis doctoral, apoyada y financiada por el programa de posgrado en Artes y Diseño de la Universidad Nacional Autónoma de México.
Fecha de recepción: 28/01/2021

Fecha de aceptación: 7/06/2021

Cómo citar: Chávez, Ch. (2021).

Diseño y sistemas complejos: un enfoque multidimensional en el proceso de Diseño RChD: creación y pensamiento, 6(10), 1-16. https//doi.org/10.5354/0719-837X.2021.60895

Revista Chilena de Diseño,

RChD: creación y pensamiento

Universidad de Chile

2021, 6(10).

http://rchd.uchile.cl

\section{Diseño y sistemas complejos: un enfoque multidimensional en el proceso de Diseño}

\author{
Design and Complex Systems: A Multidimensional Approach \\ to the Design Process
}

Resumen. Adoptar un enfoque multidimensional del Diseño, en la búsqueda de la transformación social y ecológica, supone un cambio sustancial respecto a la manera habitual de concebir su naturaleza. En este sentido, la teoría de los sistemas complejos ha dejado de orbitar en torno a las ciencias duras o sociales y ha comenzado a entrar en el campo del Diseño. Este artículo explora y discute la relación entre el Diseño y los sistemas complejos, lo que nos lleva a reconsiderar el valor y las limitaciones que ofrece para la enseñanza y la práctica del Diseño. Se argumenta que una forma de demostrar las aportaciones y la importancia de este enfoque es integrar los principios de los sistemas complejos en el proceso de diseño. Este artículo se divide en dos partes principales. En primer lugar, presento los argumentos teóricos relativos a la noción de complejidad, sistemas, problemas y pensamiento sistémico que justifican la transición a un cambio de enfoque epistemológico. A continuación, resumo los criterios e implicaciones que contribuyen a la comprensión sistémica en el Proceso de Diseño. El alcance de este manuscrito contiene reflexiones derivadas de mi investigación doctoral, en la que se propone la teoría de los sistemas complejos como una posibilidad para abordar los problemas en el Diseño del mundo contemporáneo.

Palabras clave: Diseño, enfoque multidimensional, epistemología, proceso de Diseño, sistemas complejos

\begin{abstract}
Adopting a multidimensional approach to Design, in the search for social and ecological transformation, implies a substantial change concerning the usual way of conceiving its nature. In this sense, the theory of complex systems has ceased to orbit around the hard or social sciences and has begun to enter the field of Design. This article provides an exploration and discussion of the relationship between Design and complex systems, which leads us to reconsider the value and limitations it offers for the teaching and practice of Design. It is argued that one way to demonstrate the contributions and importance of this approach is to integrate the principles of complex systems into the Design Process. This paper is divided into two main parts. First, I present theoretical arguments regarding the notion of complexity, systems, wicked problems, and systems thinking that justify the transition to a change of epistemological approach. Then, I summarize the criteria and implications that contribute to systemic understanding in the Design Process. The scope of this manuscript contains reflections derived from my doctoral research, in which complex systems theory is proposed as a possibility for addressing problems in contemporary world Design.
\end{abstract}

Keywords: Design, multidimensional approach, epistemology, Design process, complex systems 


\section{Introducción}

Históricamente, la naturaleza del Diseño (entendida como el conjunto de cualidades y características que le son propias) ha centrado sus métodos en el hacer, es decir, en la producción y la forma de los objetos. Esto debido a que el Diseño "es un campo que surge de la práctica de oficios y por lo tanto su relación con la capacidad de hacer, es parte de su origen y por lo tanto, se espera que el conocimiento que lo alimenta sea de naturaleza tal que apoye esta fundamental orientación" (Rodríguez, 2018, p. 144). En tal sentido, la palabra Diseño en castellano tiene todavía un significado limitado en cuanto a su aspecto formal y estético, ya que frecuentemente hace referencia a la representación de trazos, signos gráficos o dibujos; de ahí que su significado se vincule con las características externas de un objeto de Diseño como representación, forma, tamaño, dimensión, textura, color, etc. No obstante, la disciplina presenta una clara expansión en sus campos de acción y, sus implicaciones en el contexto real, que podría evolucionar hacia una nuevo paradigma donde se hace evidente la transición sistémica.

Dicho de otro modo, este campo atraviesa por un período de transformación epistemológica que genera una nueva reflexión sobre su sentido y función, respecto a otras maneras de pensar y diseñar frente a un mundo complejo, lleno de incertidumbres y necesidades emergentes. Es necesario reflexionar más sobre las relaciones entre el concebir y el proyectar, es decir, entre el sentir, pensar y el hacer, lo que supone la proximidad de un conocimiento más profundo con los otros (con el mundo en sí). Se requiere pasar de la mirada del diseñador que produce, a la de un pensador sistémico y estratégico que sea capaz de plantear soluciones innovadoras desde una perspectiva multidimensional. Se infiere que una manera de lograrlo es a través del estudio y entendimiento de los sistemas complejos.

De acuerdo con Pourdehnad, Wexler y Wilson (2011), la forma en que abordamos las condiciones problemáticas emergentes depende de la calidad de los enfoques que utilizamos y tratamos de aplicar. La aportación de estos enfoques requiere de una modificación de pensamiento o visión del mundo más que del uso de la tecnología. Así, la práctica de Diseño se beneficiará con la integración del enfoque de sistemas complejos en su metodología, debido a la necesidad de un cambio de enfoque epistemológico. Se hace evidente que los métodos de Diseño tradicionales para planificar y crear estrategias de respuesta son muchas veces inadecuados para abordar los retos del siglo XXI.

Los métodos y estrategias empleadas para diseñar, son modelos que encajan en los paradigmas tradicionales de la creación y transformación de objetos. Los nuevos paradigmas del diseño, exigen nuevas perspectivas teóricas que den pautas eficaces en el quehacer de designar. Estrategias basadas en el estudio y comportamiento de los patrones $y$ leyes que rigen a los sistemas emergentes (Sosa, 2017, p. 36).

\section{La noción de complejidad y sistemas complejos}

Parte del lenguaje que hoy se utiliza cuando se habla de situaciones complejas, específicamente cuando se habla de sostenibilidad o problemáticas socio-ecológicas, se hace referencia a la noción de sistemas 
y complejidad. Muchas veces no queda claro a qué se refieren estos conceptos y, de cierto modo, tampoco es explícito el entendimiento sobre su aportación práctica. Para apreciar y potenciar la teoría de los sistemas complejos y comprender de qué manera puede favorecer a la disciplina del Diseño, resulta necesario investigar ¿Cómo tratar de estudiar los sistemas sobre los que se inserta el Diseño (sistemas de personas y de naturaleza donde interaccionan)? ¿Cómo pueden ser manejados de la mejor forma posible para garantizar un cambio de enfoque en el proceso de Diseño? con el fin de reconocer las potencialidades o limitaciones que ofrece. Existen aportaciones teóricas que permiten observar esta transición sistémica como una estrategia para contribuir a una reforma de pensamiento y acción acorde a la realidad actual.

Cabe señalar que la construcción de la complejidad como problema científico emerge en las décadas de 1940 y 1950 cuando surgen las primeras teorías, conceptos y métodos para abordarlos. Weaver en su artículo "Science and Complexity" (1948) definió un nuevo tipo de problemas de complejidad desorganizada para referirse a aquellos fenómenos donde intervienen un número amplio de factores o variables interrelacionados que conforman un todo orgánico, estudiados a partir de modelos estadísticos. No es exagerado decir que, en el texto de Weaver, el significante complejidad irrumpe en el vocabulario científico, lo que permitirá décadas más tarde, dotar de identidad, a un conjunto de teorías formuladas en distintas disciplinas y ciencias (Rodríguez Zoya \& Aguirre, 2011).

Desde los tiempos de Weaver hasta el presente, el estudio de la complejidad y sistemas complejos se ha desarrollado de modo vigoroso como un campo de investigación innovador y altamente dinámico de la ciencia contemporánea. Muestra de ello, son los diversos centros creados para su estudio en las principales universidades alrededor del mundo. A su vez, la teoría de la complejidad ha sido desarrollada por autores como Prigogine (1977), Maturana y Varela (1972), Bateson (1991), Capra (1998) y Morin (1999). Hoy ya no se puede pensar en la transformación de la naturaleza y humanidad, sin tomar en cuenta estos descubrimientos que se iniciaron con la cibernética, epistemología genética, sistemas adaptativos y autopoiéticos, ciencias de la comunicación y organizativas, teoría del caos y fractales.

Por otra parte, la oficialización de la noción de complejidad se inicia en el Instituto de Santa Fe (Estados Unidos) en 1984. Este instituto se dedica a los sistemas complejos, donde hay muchas interacciones, retroacciones y variaciones (difíciles de entender con la visión clásica), donde la complejidad surge cuando muchos agentes interactúan y se adaptan entre sí y a su entorno. Algunos ejemplos de estos sistemas complejos son el sistema nervioso, Internet, los ecosistemas, las economías, las ciudades y las civilizaciones (Santa Fe Institute, 2021). En este sentido, los modelos sistémicos, procuran organizar la complejidad y describir los diferentes aspectos de los sistemas.

Para fines de este artículo, entenderemos la complejidad como el estudio que explica el comportamiento de un fenómeno natural o social desde la perspectiva de la totalidad. En otras palabras "la complejidad corresponde 
Figura 1. Componentes de un sistema (respecto al sistema-Diseño) donde se observa la organización; incluye las relaciones entre los subsistemas y las interacciones, con base en la descripción de García, 2006 (elaboración propia)

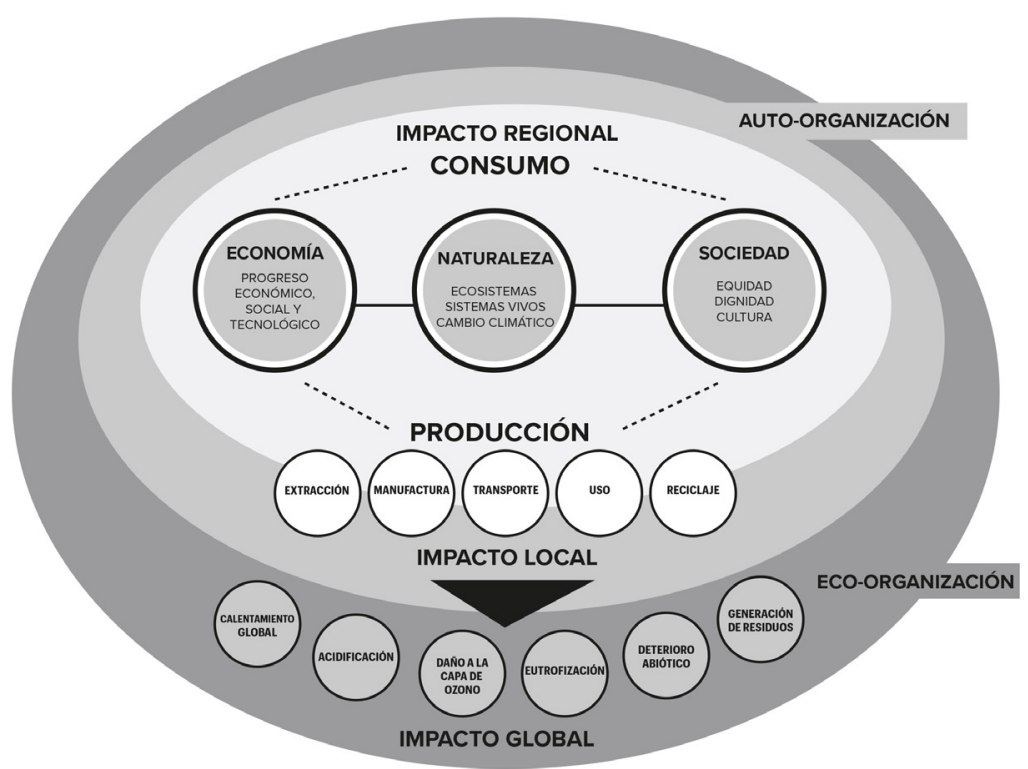

al grado de libertad de un sistema determinado" (Maldonado \& Cruz, 2011, p. 24) donde la dimensión de sus posibilidades y sus articulaciones se condensan en conceptos como adaptación, emergencia, interacción, organización y creatividad, entre otros.

En el estudio de los sistemas complejos, la cuestión central es entender cómo se produce ese comportamiento emergente y autoorganizado (Mitchell, 2009). Investigar un sistema complejo significa estudiar un trozo de la realidad que incluye aspectos físicos, biológicos, sociales, económicos, políticos (García, 2006). Los sistemas complejos son un campo de investigación inter y transdisciplinar que trata de explicar cómo un gran número de entidades relativamente simples se organizan en un todo colectivo que crea patrones, utiliza información y, en algunos casos, evoluciona y aprende.

Bajo este panorama se puede añadir que todo Diseño es un sistema (Buchanan, 2019). En la organización de un sistema-Diseño (ver Figura ı) se encuentra el conjunto de relaciones entre los elementos: factores que actúan directa o indirectamente, subsistemas que corresponden a cierto tipo de procesos agrupables con mayor grado de interconexión, flujos de entradas y salidas que suelen ser materia, energía, información, recursos o políticas y las condiciones de contorno o límites que son el conjunto de tales interacciones que ejercen influencia sobre un nivel dado (García, 2006). Todo sistema está situado dentro de un cierto entorno, ambiente o contexto, que lo circunda, lo rodea o lo envuelve total y absolutamente (Moriello, 2016).

Para describir adecuadamente su comportamiento, es necesario conocer su organización, es decir, la disposición de sus elementos componentes (parte espacial-estática-estructural) y las interacciones o relaciones que se establecen entre ellos (parte temporal-dinámica-funcional) (Moriello, 2016). La Figura 2 describe los componentes de un sistema complejo conforme 


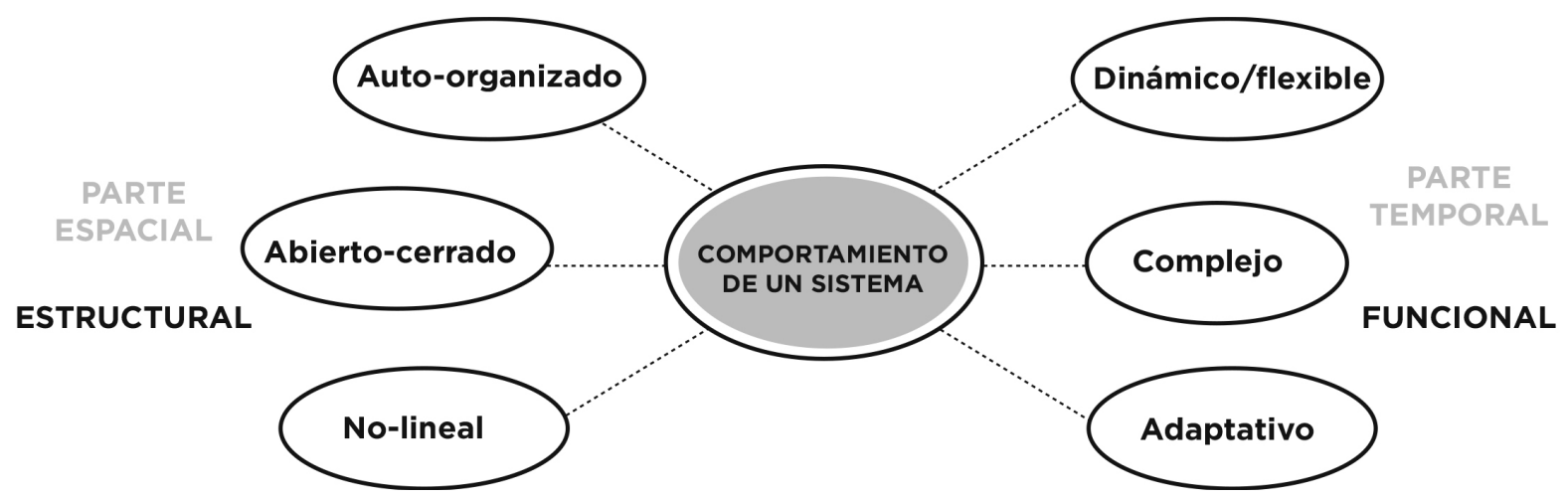

Figura 2. Disposición de los componentes de un sistema: parte espacial-estática-estructural

Pocas variables / homógeneos

Muchas variables / heterogéneos

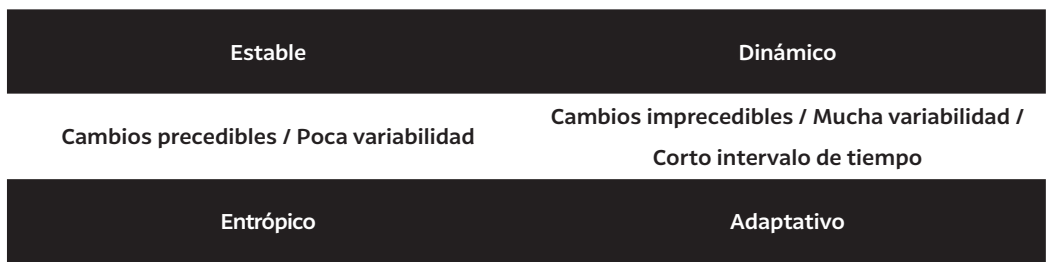

Comportamiento inesperado, no predecible

Capacidad adaptativa frente a perturbaciones o fluctuaciones del entorno

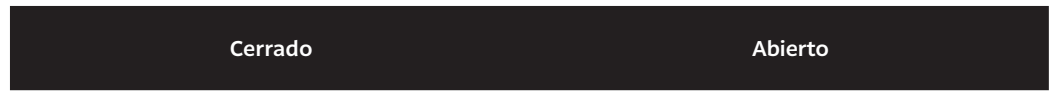

Intercambio materia / energía / información

No hay intercambio de materia con el sistema externo, pero hay un intercambio de energía de forma parcial o selectiva (incluyéndola o expulsándola) y parte temporal-dinámica-funcional, en base a Moriello, 2016 (elaboración propia)

Tabla 1. Diferenciación de características y comportamiento de un sistema, en base a Moriello, 2016 (elaboración propia)

a sus dinámicas y estructuras. Las dos dimensiones coexisten y son complementarias.

Lo que hace complejo a un sistema no es necesariamente la cantidad de variables o factores, sino cómo son las relaciones entre esos factores y las interacciones que involucra, es decir, cómo es su comportamiento o la dinámica que presenta. Por ejemplo, si el sistema es recursivo antes que determinista, abierto o cerrado, no-lineal, adaptativo o atravesado por dinámicas impredecibles, caóticas o marcadas de incertidumbre.

A continuación, en la Tabla I se pueden observar las principales diferencias entre las características en el comportamiento de un sistema, con el fin de posibilitar una taxonomía básica para el diagnóstico e identificación de un problema complejo. Más adelante se explicará cómo se conectan estos conceptos con el proceso de Diseño.

\section{Algunas aportaciones teóricas sobre los sistemas en Diseño}

En la disciplina del Diseño, el primer acercamiento que se visualiza en el estudio de los sistemas, es la propuesta realizada por Hebert Simon sobre 
el Diseño como una ciencia de lo artificial, la cual debería encargarse de enseñar lo relacionado con las cosas artificiales creadas por el ser humano. En su proposición intentó vincular la práctica del Diseño con los principios de ingeniería. Expuso que el Diseño se ocupa de cómo idear artefactos para conseguir objetivos (goal-oriented) y en la raíz de ello figura el proceso de Diseño (Simon, 2008). Señala que "diseña todo aquel que desarrolla un acto destinado a transformar una situación existente en una situación preferible" (Simon, 2008, p. 123). Cabe resaltar que Simon en The Architecture of Complexity (1962) apunta a una definición (aunque no formal) de sistemas complejos. "A grandes rasgos, entiendo por sistema complejo uno formado por un gran número de partes que interactúan de forma no simple" (Simon, 1962, p. 468). El mismo autor planteó que entender un sistema jerárquico con estructura compleja, sería una técnica poderosa para descubrir la forma viable de descomponerla en subcomponentes interrelacionados, y por lo tanto cada componente tiene cierto grado de interdependencia de los demás, ya que cada uno afectará a los demás a través de su función (Simon, 1962). Medio siglo después, algunas de estas aportaciones continúan aplicándose al Diseño de objetos.

A pesar de que esta concepción varía de lo que hoy se concibe como sistema complejo por las ciencias de la complejidad, es importante destacar que la propuesta de Simon (basada en la teoría de la jerarquía) respondía a la necesidad de sintetizar y analizar la complejidad, por lo que facilitó la información y técnicas para lograrlo. En su modelo de Diseño problem-solving señala que "la resolución de problemas requiere un proceso selectivo de ensayo y error. Cuánto más difícil y novedoso sea el problema, mayor será la cantidad de prueba y error necesaria para encontrar una solución" (Simon, 1962, p. 473). Ahora bien, la ciencia del Diseño evolucionó fuertemente hacia una orientación en los métodos del Diseño. Manifestado un pensamiento más bien sistemático, con un racionalismo limitado que después fue rechazado incluso por algunos de los teóricos originales (Jones, 2014).

El segundo acercamiento hacia una visión sistémica del Diseño, lo establecen Rittel y Webber (1973) cuando elaboraron la noción de wicked problems como un aspecto esencial que constituye la complejidad de los métodos de Diseño. Lo definen como "una clase de problemas del sistema social que están mal formulados, con información confusa y caracterizados por ser no controlables. Se trata de problemas difíciles de abordar y resolver desde planteamientos lineales clásicos o enfoques analíticos tradicionales, ya que dependen de escurridizos juicios para su llegar a su resolución" (Rittel, 1973, p. 160). En su planteamiento, encontrar un problema conlleva hallar su solución puesto que "toda especificación de un problema es una especificación del camino a seguir para tratarlo" (Rittel, 1973, p. 173).

En concordancia con Peter Jones (2014), en el caso de los problemas complejos desde una posición reciente, es incorrecto hablar de resolución de problemas, ya que no hay medidas de evaluación acordadas o efectivas que justifiquen la afirmación. Existe una fuerte tendencia en la actividad de Diseño a centrar sus métodos en las soluciones formales o materializables del problema. A pesar de que la racionalidad del Diseño es multifacética, heterogénea y multinivel, el proceso de Diseño está marcado por una brecha entre el análisis de problema y la solución del Diseño, esto es, la división 
conceptual entre necesidad y forma, por tanto, es necesario su abordaje desde un enfoque multidimensional.

El pensamiento de diseño inscrito en la resolución de problemas sistemas sociales (complejos por definición) debe tener una forma de abordaje muy específica y particular que, atendiendo múltiples aspectos provenientes de diversas disciplinas, campos del conocimiento y la experiencia humana deben interpretar y traducir en requerimientos para las soluciones de diseño (Arámbula \& Uribe, 2016, p. 171)

Hoy sabemos que la idea de resolver problemas complejos en Diseño está presente pero tiene una resonancia inadecuada y con poco significado en lo empírico. Esto debido a que en el mundo real las situaciones y los procesos no se presentan de manera que puedan ser clasificados por su correspondencia con alguna disciplina en particular. Se trata de problemáticas donde están involucrados varias disciplinas y factores como el medio físico-biológico, producción, tecnología, organización social, economía, etc. (García, 2006, 87). Los problemas complejos se caracterizan por la confluencia de múltiples procesos cuyas interrelaciones constituyen la estructura de un sistema que funciona como una totalidad organizada, denominada sistema complejo (García, 2006), y no así por su grado de dificultad para ser resueltos como lo atribuyen en su momento Simon (1962) y Rittel (1973).

Los problemas son complejos de tres maneras. Son dinámicamente complejos, cuando la causa y el efecto están muy lejos en tiempo y espacio, lo cual dificulta captarlos a primera vista. Son por naturaleza complejos, cuando se desenvuelven de manera desconocida e impredecible. Y son socialmente complejos cuando las personas involucradas ven las cosas de manera diferente, y por ello los problemas se polarizan y atascan (Kahane, 2005, p. 6)

En tal sentido, un problema complejo sólo puede ser resuelto de manera pacífica -y añado aquí, pertinente- si las personas involucradas trabajan juntas, con ánimo creativo, para entender su situación y mejorarla.

"Debemos aprender a hacer las cosas de otra manera, menos común y más abierta" (Kahane, 2005, p. 7). Igualmente, se infiere que los problemas complejos son caracterizados así porque pueden mostrar cambios a lo largo del tiempo, variabilidad e incertidumbre, lo que puede dar lugar a diferencias entre las partes interesadas o modificaciones sustanciales en los límites del problema inicial.

Para seguir con el análisis, un tercer acercamiento lo señala Richard Buchanan en su artículo "Wicked Problems in Design Thinking" (1992) donde expone el surgimiento de un cuarto ámbito del Diseño "entornos complejos" (ver Figura 3) así como las diferentes formulaciones y ampliaciones de la solución de problemas, incluida la integración sistémica de los seres humanos en entornos ecológicos y culturales más amplios, la cual se describe como: "la exploración del rol del diseño para sostener, desarrollar e integrar al ser humano a ambientes ecológicos y culturales, adaptándolos para que sean deseables y sobre todo necesarios" (Buchanan, 1992, p. 20). Los cuatro ejes de acción tienen en común poner en énfasis la experiencia 
Figura 3. Las cuatro órdenes del Diseño propuestas por Richard Buchanan en 1992, de acuerdo a la ampliación de sus ejes de acción (elaboración propia)

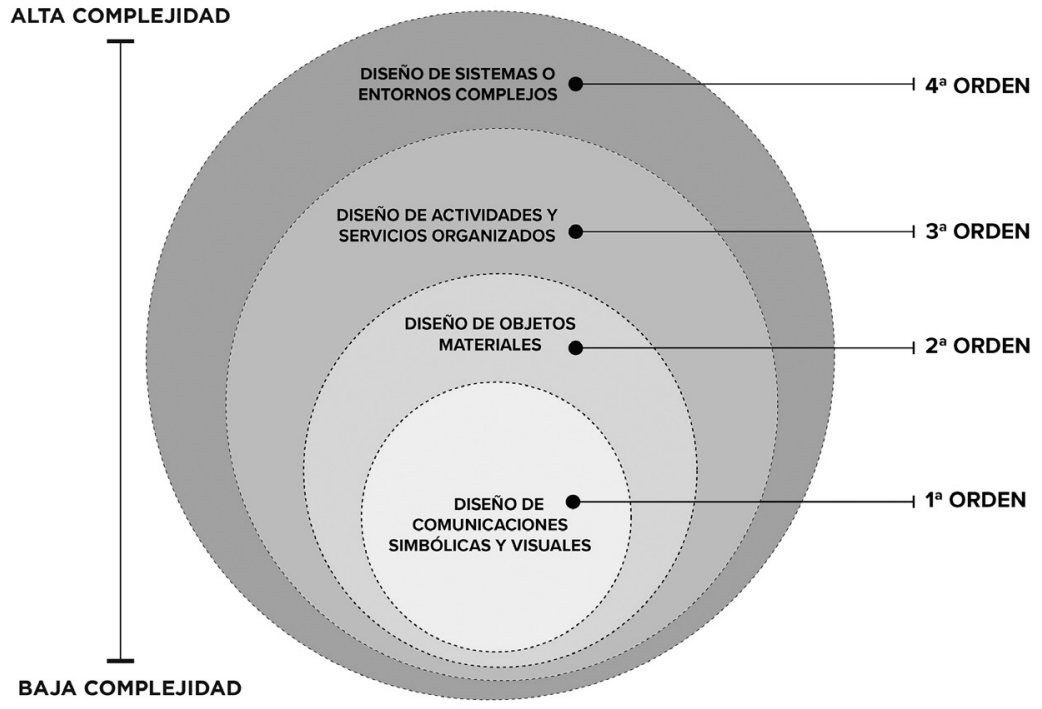

humana en la cultura del Diseño actual. Esto también evidencia la transición hacia una disciplina más integrativa y los esfuerzos epistemológicos por alcanzarlo, al integrar los conocimientos de nuevas maneras, adaptadas a circunstancias y necesidades específicas del contexto.

De igual modo, Ezio Manzini (2015) ha destacado la idea de los sistemas complejos para tener injerencia en la experiencia humana, en la creación de sistemas y ciudades sostenible que permitan la diversidad y cohesión social, así como generar puentes para abonar la conectividad, colaboración y resiliencia, definida ésta última como "una forma de diversidad, redundancia y experimentación continua" (Manzini, 2015, p. 29). Puntualiza la importancia de crear redes que incluyan el intercambio de conocimiento diverso, "para generar la capacidad de impacto en la sociedad es necesario un cambio radical, uno que requiere una discontinuidad sistémica" (Manzini, 2015, p. 29) y afirma que "el diseño, más allá de buscan dar soluciones a problemas inmediatos, debería convertirse en un agente de cambio social” (Manzini, 2015, p. 5).

A tal efecto, actualmente las organizaciones buscan potencializar la creatividad y la imaginación colectiva. Con estos cambios, viene un nuevo conjunto de enfoques centrados en la comprensión de sistemas abiertos, interrelacionados y medios de participación activa.

Aunque existen otros ejemplos de teóricos de Diseño contemporáneos que abordan el enfoque sistémico y su incidencia en el Diseño, como William McDonough y Michel Braungart (1992) Alain Findeli (2001), Jorge Frascara (2002), Victor Margolin (2002), John Thackara (2005) y Kees Dorst (2015), aún es carente la información respecto a su operatividad en el proceso de Diseño, lo cual posibilita realizar una introspección más profunda sobre los alcances, límites y significado de la práctica actual. Como hallazgos, a partir de la revisión de literatura, se han identificado algunos criterios que aportan los sistemas complejos en los métodos de Diseño, tendencias de investigación académica y en la creación de modelos. 
Tal como lo indica Jones (2014b) en su artículo "Systemic Design Principles for Complex Social Systems", la teoría de los sistemas y sus directrices en la práctica se han promovido como las mejores técnicas para aumentar la conciencia social de problemas interconectados que pueden determinar el destino humano. Son pocos los estudios recientes que han definido está posición emergente. A pesar a ello, la mayoría de las ideas la reconocen como una epistemología práctica de resolución de problemas (Jones, 2014). El movimiento sistémico ha sido fuertemente criticado por ser considerado poco realista lo que ha llevado a algunos a reclamar más la integración del pensamiento sistémico con los métodos prácticos en la actividad actual.

Lo anterior significa que el pensamiento sistémico proporcionaría en los métodos de Diseño una visión global y totalizadora respecto a la redes de relaciones (imbricadas dentro de redes mayores), las cuales se interrelacionan a su vez con un contexto, entorno o medio ambiente. En palabras de Capra, el pensamiento sistémico contribuyen a la comprensión de la vida, que va de una concepción mecanicista hacia una ecológica, lo cual significa:

Una nueva perspectiva para visualizar la trama de la vida como sistemas vivos interactuando con otros sistemas; constituida por redes dentro de redes. La trama de la vida como parte de la comprensión de la naturaleza desde un punto de vista sistémico y complejo, ha contribuido a abrir otras puertas del conocimiento para que puedan seguir surgiendo nuevos enfoques alternativos, es decir, diálogos interdisciplinarios para explorar nuevas ideas y modos de pensar (Capra, 1998).

En síntesis, se presentaron algunos argumentos relevantes sobre teóricos de Diseño que actualmente han valorado el impacto epistemológico de los sistemas complejos dentro de la disciplina del Diseño. Gran parte de esta preocupación ha surgido por la necesidad de hacer visible sus aportaciones prácticas en los métodos de Diseño. Esto incluye que los diseñadores adquieran una mayor estudio y comprensión de la complejidad de los sistemas y desarrollen nuevas alternativas para diseñar en éstos la solución de problemas complejos de manera más pertinente.

\section{El proceso del diseño desde un enfoque sistémico-complejo}

Las investigaciones empíricas en el campo del Diseño y sus carencias respecto a un enfoque sistémico y complejo en sus métodos, me han conducido a tratar de aportar -sobre categorías conceptuales y procedimentales- que puedan operar en el proceso de Diseño de manera significativa, al considerar que el Diseño requiere de un pensamiento y acción que involucre un conocimiento más profundo sobre la complejidad de los problemas.

La reflexión actual sobre el proceso de Diseño, entendido como el conjunto de fases o procedimientos aplicados para llegar a un fin, solución o lograr un conocimiento específico, ha sido parte de las preocupaciones intelectuales en la teoría del Diseño y en general, se ha relacionado con la toma de decisiones. Al respecto, es necesario resaltar que los modelos de Diseño han cambiado constante y permanentemente durante varias épocas con diferentes propósitos y enfoques. A pesar de ello, dentro de la metodología de Diseño, la naturaleza de los problemas de Diseño ha estado descrita 


\section{ESTRUCTURA LINEAL}

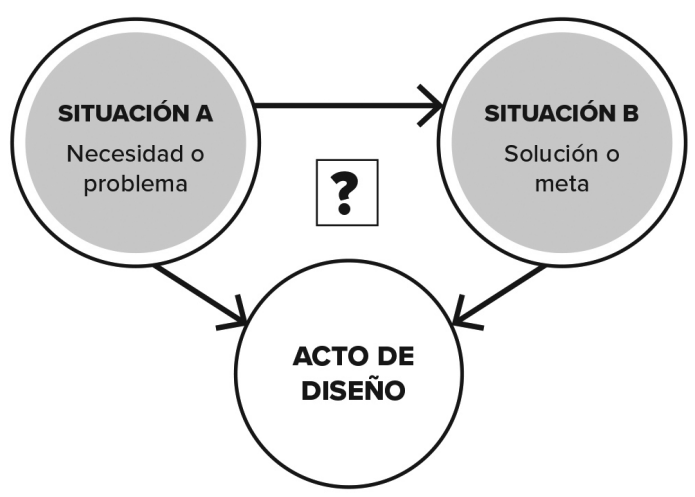

ESTRUCTURA SISTÉMICA

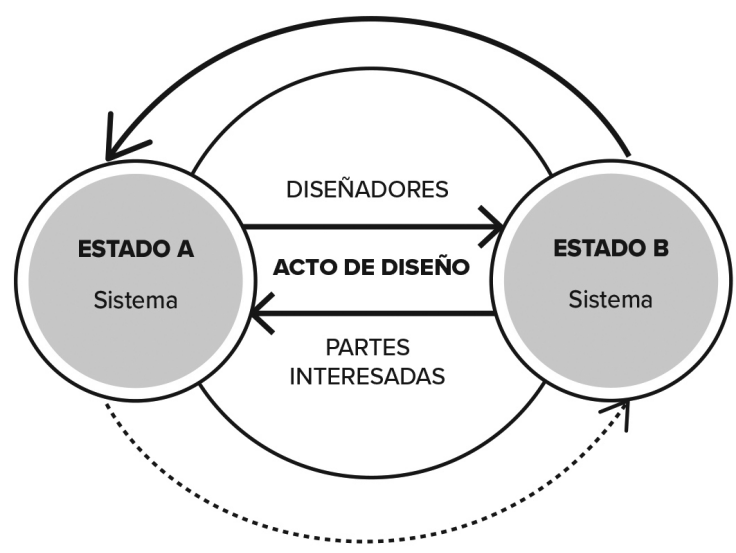

Figura 4. Diferencias entre la estructura lógicalineal del proceso de Diseño y la propuesta de una nueva estructura lógica del proceso de Diseño basada en las partes interesadas, con base en Findeli, 2001 (elaboración propia) como deficiente (Dorst, 2015), pues pareciera que el Diseño ya no tiene claridad conceptual ni metodológica, abarca varios aspectos o funciones la vida social que van desde las necesidades para el bienestar de vida hasta cuestiones para el consumo humano y el esparcimiento, con lo cual puede traer algunas desventajas en su epistemología.

De manera general, se puede considerar que la mayoría de los enfoques en los métodos de Diseño son de tipo proyectual, es decir, con un objetivo de actividad representacional simbólica (Burgos, 2016). La metodología del Diseño ha tenido un recorrido largo, aunque a veces ausente en cuanto a la estructura de las representaciones lógicas para el análisis del problema del Diseño, ya que el foco ha estado centrado en las etapas del proceso.

Al respecto, Alain Findeli (2001) afirma que como resultado de las diversas crisis paradigmáticas en las escuelas de Diseño todavía a menudo se suele decir que si el problema está bien planteado (es decir, si la investigación preliminar se ha llevado a cabo a fondo y los criterios funcionales se han establecido con precisión de acuerdo a la funciones del objeto), la solución se conseguirá casi automáticamente. Esta es "la estructura lógica más ampliamente aceptada y de alguna manera más practicada en el proceso de diseño" (Findeli, 2001, p. 10) (ver Figura 4). El autor puntualiza que se debe adoptar la idea de sistema desde el inicio del proceso:

1. En lugar de un problema, tenemos: estado A de un sistema;

2. En lugar de una solución, tenemos: estado B del sistema; y

3. El diseñador y el usuario forman parte del sistema (partes interesadas).

La proposición de Findeli (2001), a simple vista puede parecer no tan sustancial. Sin embargo, la idea de modificar una estructura lineal hacia una nueva estructura lógica del acto de Diseño desde las partes interesadas, representa un cambio epistemológico potencial donde será posible encontrar nuevos caminos para proyectar. A partir de la integración sistémica, se pueden detectar otros elementos significativos en las distintas fases de Diseño, así como valorar las dinámicas de retroalimentación continua dentro de un proceso abierto, iterativo y participativo. Esto supone un impacto directo en la 


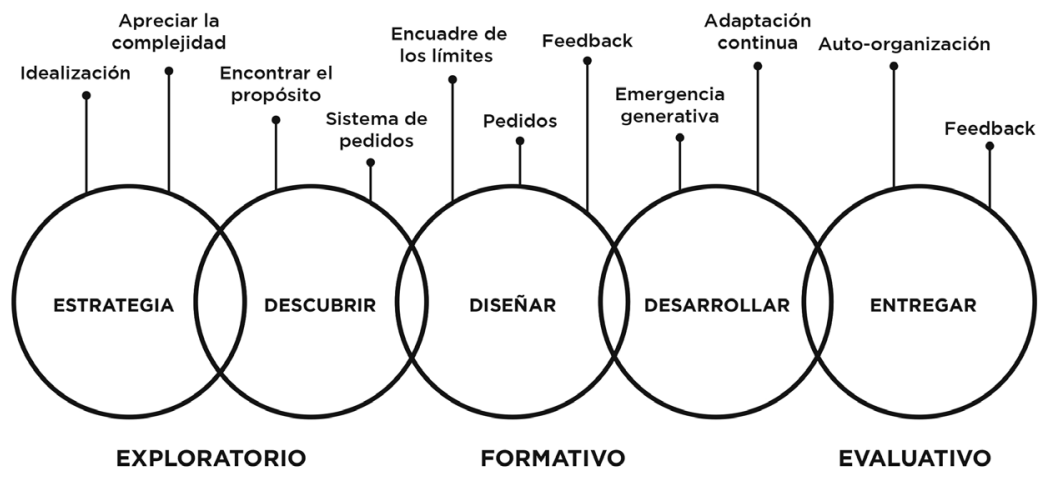

enseñanza del Diseño y, por consiguiente, en las competencias y habilidades necesarias de los futuros profesionales de Diseño.

Por su parte, Jones formula de manera conjunta, aunque desde su punto de vista incompleta, algunos principios de Diseño sistémico donde examina sus correspondencias, que se basan en el metaanálisis de conceptos seleccionados para la resolución de problemas complejos. Algunas de estas etapas son: idealización, apreciar la complejidad, encontrar el propósito, encuadre de los límites, la variedad necesaria, retroalimentación, el sistema de pedidos, emergencia generativa, adaptación continua, auto-organización (Jones, 2014) (ver Figura 5).

Una aportación de este modelo es que la visualización de la proliferación de intervenciones que puede tener el diseñador frente a las necesidades u objetivos del problema y ante la diversidad de posibles explicaciones o abordajes para su solución. Esto pone en relieve el papel de generar una variedad de soluciones precisamente como un medio de análisis del problema, pero también es importante entender su estructura donde se contemple la dinámica del sistema, las interacciones de los elementos y las partes interesadas. En tal sentido, la teoría de los sistemas complejos ha comenzado a trazar otras oportunidades para responder a estos desafíos emergentes en la práctica de Diseño en un entorno cada vez más cambiante.

\section{Resultados y discusión}

Podemos condensar lo dicho hasta aquí que en los desafíos de la sociedad contemporánea se plantea el significado de lo complejo y lo sistémico como dos posibilidades de pensamiento hacia un mundo más habitable, el cual no consiste simplemente en crear más productos ecológicos para el consumo, sino en repensar, visualizar o gestionar procesos y métodos que promuevan un comportamiento responsable generalizado encaminado hacia el cambio social. Para la propuesta de aproximación a un enfoque multidimensional se presentaron los principales conceptos y características relacionadas con los sistemas complejos, con la idea de proponer un nuevo lenguaje para el Diseño
Figura 5. Principios de Diseño asignados al modelo de Diseño (Design Thinking) propuesto por Jones, 2014b (elaboración propia) 
Figura 6. Propuesta de una cosmovisión sistémica-compleja en el Diseño (elaboración propia)

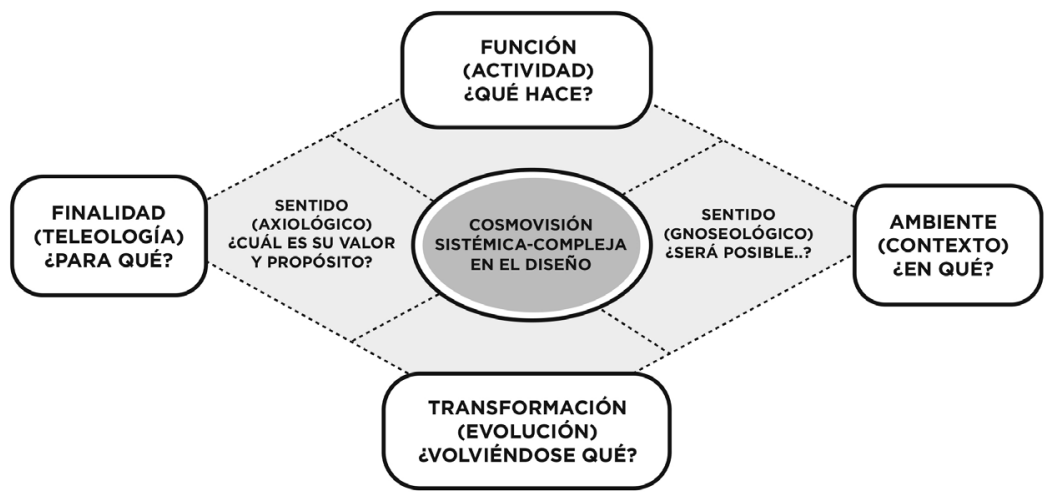

desde autores como García (2006) y Moriello (2016), así como las aportaciones metodológicas realizadas al proceso de Diseño (Jones, 2014) (Findeli, 2001) para plantear su posible integración en la epistemología del Diseño.

El propósito es establecer que la racionalidad de Diseño -en su extensa complejidad y multiplicidad de elementos- debería asumir esa corresponsabilidad desde una visión ampliada; esto es transdisciplinaria, colaborativa, empática, sensible, crítica, ética, pero también como una disciplina compleja, abierta, divergente, flexible y en permanente evolución.

Las metodologías de Diseño para la planificar proyectos requieren comprender las condiciones emergentes del entorno. De modo que, a partir de la comprensión de un fenómeno de estudio o problema entendido como sistema complejo, se ayudará a identificar y evaluar ciertas circunstancias que rodean a los fenómenos para producir un impacto significativo y formular o ejecutar estrategias de forma idónea. A continuación, se propone considerar algunas preguntas clave en el proceso de Diseño para el análisis sistémico.

1. ¿Qué elementos en su sistema hablarían de su complejidad?

2. ¿Qué elementos en su sistema hablarían de su adaptabilidad?

3. ¿Qué elementos en su sistema hablarían de su dinámica y estabilidad?

4. ¿Qué elementos en su sistema hablarían de su entropía?

5. ¿Qué perturbaciones y tensiones irritarían a su sistema?

Estas preguntas pueden facilitar el abordaje del fenómeno Diseño como sistema complejo, lo cual permitirá aumentar un pensamiento crítico y sistémico los diseñadores respecto a la toma de decisiones, lo cual significa considerar todos los actores y factores que lo construyen, es decir, adquirir un enfoque multidimensional desde la concepción del proyecto. A tal efecto, a continuación, se señalan tres puntos importantes como alternativas o rutas que pueden favorecer la integración del enfoque sistémico en el proceso de Diseño.

\section{Integrar la cosmovisión sistémica-compleja}

Este enfoque plantea varios desafíos para la producción de conocimiento en la disciplina, lo cual implica un reto mayor, la adición de nuevas perspectivas en el entendimiento de la relación Diseño-contexto. Se trata de buscar respuestas a preguntas basadas no sólo en la función de los objetos (qué hace), sino ampliar a una visión sistémica a través de cuestionamientos 


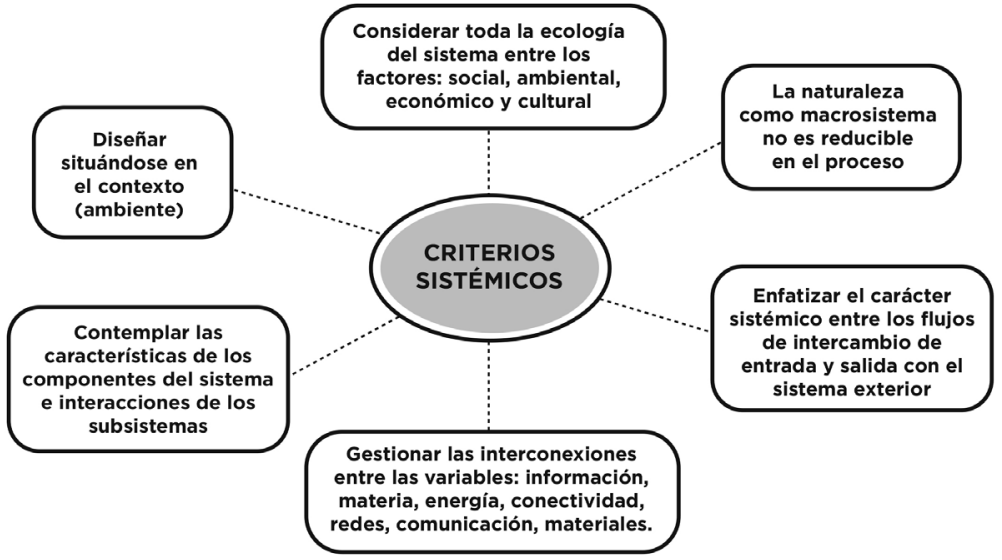

más reflexivos: para qué, en qué o volviéndose qué; pensar en la idea de un objeto-sistema que involucra pensar en el entorno, en una finalidad, en una actividad, y en las consecuencias o transformaciones que tendrá dicha actividad. Es una nueva cosmovisión sistémica-compleja del Diseño (ver Figura 6). La organización actual del sistema-Diseño conlleva el surgimiento de problemas fundamentales que afectan la vida humana, por tanto, se vuelve vital pensar críticamente en las implicaciones sociales, políticas, ambientales, antropológicas y humanas de la actividad. La epistemológica práctica del Diseño tendrá que adquirir una nueva dimensión.

El desafío para la práctica es que exista un mayor acercamiento entre la relación Diseño-naturaleza-sociedad que permita definir esquemas dinámicos para su aplicación sistémica en el contexto, no sólo de manera ergonómica, estética o funcional. El Diseño ya no debería asumirse parcialmente sino con un impacto local-global (macro-sistémico), lo cual implica la búsqueda de soluciones e innovaciones que favorezcan a esta integración.

\section{Establecer criterios sistémicos en el proceso de Diseño} La naturaleza siempre cambiante del Diseño, las modificaciones y transformaciones que debe asumir para contextualizar su accionar, la pluralidad de los discursos y las fuertes presiones del entorno, nos conducen a pensar en la necesidad de comprender estos "sistemas resilientes, que son, en su esencia, sistemas adaptativos, complejos, cargados de incertidumbre, pero con alta capacidad de respuesta y recuperación" (Malo, 2016, p. 140). Se propone entonces un conjunto básico de criterios sistémicos (ver Figura 7) que puedan ser considerados en el proceso de Diseño, situándose en el contexto y no en la forma del objeto, de tal modo que se facilite la operacionalización sistémica.

\section{Construir un conocimiento común a partir de la integración sistémica} Para lograr una integración sistémica, en la concepción de los sistemas complejos (García, 2006) es importante incluir -desde la formulación del problema y la pregunta conductora- la relación entre el objeto de estudio y las disciplinas a partir de las cuales se realiza el estudio, y no sólo tomar en cuenta los aspectos particulares o características de un fenómeno, proceso o situación. Es a partir del trabajo inter y transdisciplinario que se obtiene una visión en común que integra el conocimiento disponible entre los diferentes actores. Esto incluye a los diseñadores, científicos, políticos, empresarios y
Figura 7. Propuesta de criterios sistémicos en el proceso de diseño para la operacionalización sistémica (elaboración propia) 
Figura 8. Integración sistémica entre las partes interesadas, a partir de la propuesta de García, 2006 (elaboración propia)

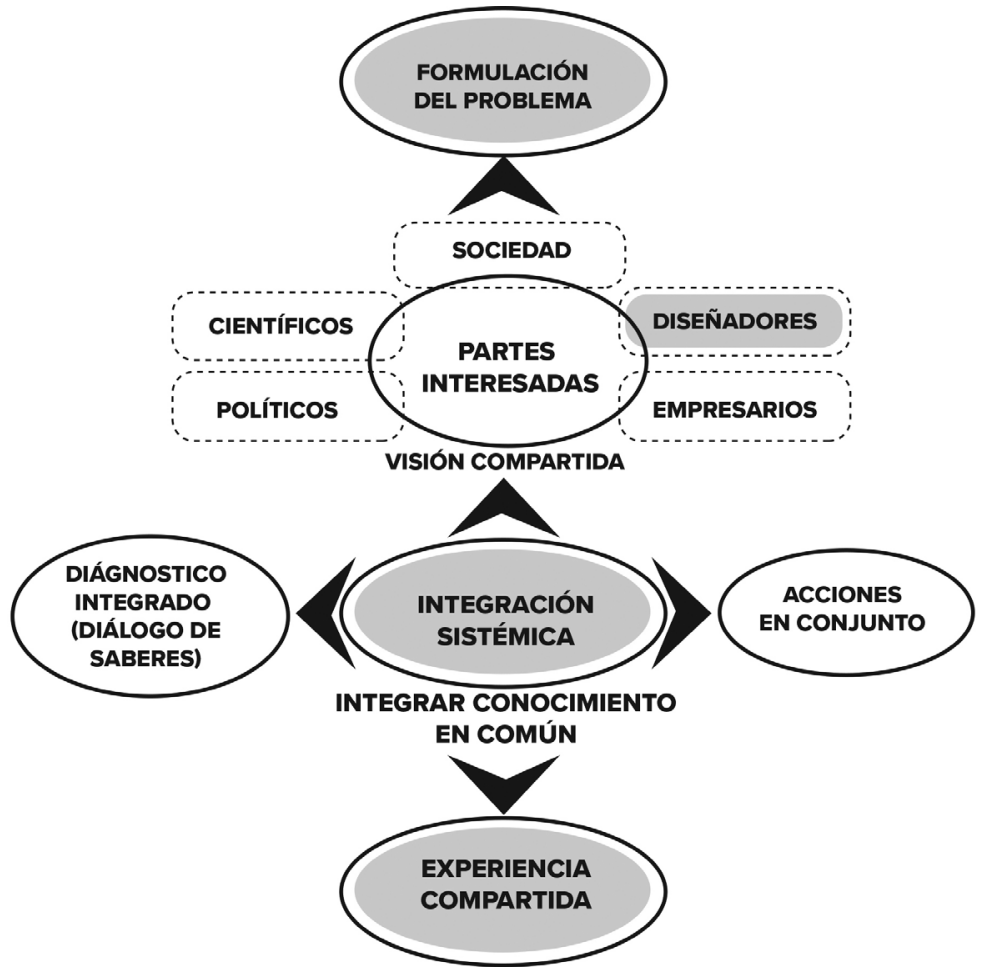

sociedad en general, lo cual contribuye a poner en marcha acciones conjuntas desde un enfoque multidimensional (ver Figura 8). Reconocer la pluralidad de saberes y diversidad de causalidades de un problema irreductible posibilita el proceso de aprender de la experiencia compartida. Lo que aporta el enfoque de los sistemas complejos es la aspiración a un conocimiento no parcelado de la realidad, que al mismo tiempo sea capaz de dialogar con la multiplicidad de los saberes humanos.

\section{Reflexiones}

La racionalidad del Diseño se ha modificado en sus diferentes niveles de actuación, para afrontar nuevos retos ante los acelerados cambios del mundo. A pesar de que la disciplina se encamina a una relación más estrecha entre la teoría y práctica hacia la transformación socio-ecológica de la humanidad, aún se encuentra en proceso de transición sobre su propia naturaleza y el rol que hoy juega en la cultura. La situación actual exige nuevos marcos de acción basados en una perspectiva integradora. En este sentido, una de las rutas que puede tomar el Diseño, además de comprender cómo operan los sistemas vivos, es retomar los principios de los sistemas complejos para procurar incorporar estos conceptos en el proceso creativo, ya sea como metáforas para la comprensión y explicación de una realidad o como mecanismos operativos-metodológicos para la creación de objetos.

La relevancia de problematizar esta teoría en la disciplina del Diseño es poder brindar un marco conceptual que permita replantear y generar nuevas formas de pensamiento hacia mejores prácticas. Además de ampliar la reflexión 
y discusión sobre los desafíos epistemológicos del Diseño, donde se deja entrever la posible consolidación de un entendimiento más accesible sobre los sistemas complejos con el fin de facilitar su operatividad en el proceso de Diseño. Esto sólo será asequible si se adopta una visión sistémica-compleja antes de transformar el entorno. El enfoque multidimensional que se presenta aquí puede contribuir a superar algunas dicotomías planteadas por los sistemas tradicionales para lo cual se adopta una actitud abierta, capaz de dar respuestas flexibles y creativas a la diversidad de problemas planteados en el campo del Diseño.

La disciplina de Diseño no sólo requiere ser una actividad de profundización práctica, teórica o reflexiva, sino ampliar sus horizontes disciplinarios. Algunas de estas visiones se pueden observar en cómo los límites del Diseño se han expandido hacia otros campos de conocimiento, donde el diálogo de saberes se torna imprescindible. Dentro de la propuesta de un enfoque multidimensional, es posible la fundamentación y construcción de nuevas posibilidades de respuesta en diversos sectores. Tener en cuenta un conocimiento existente sobre las nociones de sistema y complejidad, genera inevitablemente otras categorías de análisis que pueden ser inherentes en los métodos de Diseño actual que conducirán a la aplicación de estrategias integrativas, gracias a la intervención y función transformadora del Diseño.

En consecuencia, el enfoque de los sistemas complejos ha contribuido a una transformación radical del modelo mecanicista de los métodos de Diseño. La profundidad de esos descubrimientos va más allá de sus claras manifestaciones científicas y técnicas, incluye nuevas formas de pensar y actuar, que a su vez comprenden otras teorías o enfoques de pensamiento. Por lo tanto, adoptar un enfoque multidimensional desde el Diseño supone que antes de transformar está comprender su complejidad. 


\section{Referencias}

Arámbula P. \& Uribe B. (2016). Entendiendo el proceso de diseño desde la complejidad. En Kepes, 13(13), 171-195. https://doi.org/10.17151/kepes.2016.13.13.9

ASALE, R., \& RAE. (2021). Diseño/Diccionario de la lengua Española. «Diccionario de la lengua española» - Edición del Tricentenario. Recuperado el 6 de junio de 2021, de https://dle.rae.es/diseño

Bateson, G. (1991). Pasos hacia una ecología de la mente. Planeta. Buchanan, R. (1992). Wicked Problems in Design Thinking. En Design Issues, 8(2), 5-21. https://doi.org/10.2307/1511637

Buchanan, R. (2019). Systems Thinking and Design Thinking: The Search for Principles in the World We Are Making. En She Ji: The Journal of Design, Economics, and Innovation, 5(2), 85-104. https://doi.org/10.1016/j. sheji.2019.04.001

Burgos, C. (2016). Teoría del diseño: Categorías y enfoques epistémicos para una nueva imagen de la disciplina. En Revista Pensum, 2(2), 25-40.

Capra, F. (1998). La trama de la vida. Anagrama.

Dorst, K. (2015). Frame innovation: Create new thinking by design. The MIT Press.

Findeli, A. (2001). Rethinking Design Education for the 21st Century: Theoretical, Methodological, and Ethical Discussion. En Design Issues, 17(1), 5-17. https://doi. org/10.1162/07479360152103796

Frascara, J. (2002). People-centered design: complexities and uncertainties. En Design and the Social Sciences: Making connections. Taylor \& Francis

García, R. (2006). Sistemas complejos: Conceptos, métodos y fundamentación epistemológica de la investigación interdisciplinaria. Gedisa.

Jones, P. (2014). Design Research Methods in Systemic Design. En Proceedings of RSD3, Third Symposium of Relating Systems Thinking to Design. Oslo School of Architecture and Design.

Jones, P. (2014b). Systemic Design Principles for Complex Social Systems. En G. S. Metcalf(Ed.), Social Systems and Design, Vol. 1 (pp. 91-128). Springer Japan. https://doi. org/10.1007/978-4-431-54478-4_4

Kahane, A. (2005). Cómo resolver problemas complejos: Una manera abierta de hablar, escuchary crear nuevas realidades. Norma.

Maldonado, C. E. \& Gómez-Cruz, N. A. (2011). El Mundo de las Ciencias de la Complejidad: Una Investigación sobre Qué Son, Su Desarrollo y Sus Posibilidades. Universidad del Rosario. https://doi.org/10.13140/RG.2.1.4543.5286

Malo, G. (2016). Diseño Sustentable. Entre sustentos y desafíos: Por los caminos de la resiliencia y la entropía. En Revista DAYA. Diseño, Arte y Arquitectura (1). Universidad del Azuay. https://doi.org/10.33324/daya.vıiı
Matura, H. \& Varela F. (1972). El árbol de conocimiento. De las bases biológicas al entendimiento humano. Buenos Aires, Argentina: Lumen.

McDonough W. \& Braungart M. (2002). Cradle to Cradle. Remaking the Way We Make Things. North Point Press.

Mitchell, M. (2009). Complexity: A guided tour. Oxford University Press.

Moriello, S. (2016). Dinámica de los sistemas complejos. En la Comunidad del Pensamiento Complejo. https://www. pensamientocomplejo.com.ar

Margolin, V. (2002). Las políticas de lo artificial. Designio.

Manzini, E. (2015). Design, When Everybody Designs. An introduction to Design for Social Innovation. The MIT Press.

Morin, E. (1999). La cabeza bien puesta. Repensar la reforma. Reformar el pensamiento. Ediciones Nueva Visión SAIC.

Pourdehnad, J., Wexler, E. R. \& Wilson, D. V. (2011). Systems \& Design Thinking: A Conceptual Framework for Their Integration. En Organizational Dynamics Working. http:// repository.upenn.edu/od_working_papers/10

Prigogine, I. (1998). El nacimiento del tiempo. TusQuets Editores.

Prigogine I. \& Nicolis G. (1977). Self-Organization In NonEquilibrium Systems. En Society for Industrial and Applied Mathematics, 24(4), 483-485. https://www.jstor.org/ stable/2029552

Rittel, H. W. J. \& Webber, M. M. (1973). Dilemmas in a general theory of planning. En Policy Sciences, 4, 155-169.

Rodríguez, L. (2018). Diseño: Investigación y Sociedad del Conocimiento. En Nuevas vanguardias y tendencias en el diseño. Facultad del Hábitat. Universidad Autónoma de San Luis Potosí.

Santa Fe Institute. (2021). Recuperado el 6 de junio de 2021, de https://www.santafe.edu/

Simon, H. A. (1962). The Architecture of Complexity. En Proceedings of the American Philosophical Society, Vol. 106, Num. 6 (pp. 467-482). http://www.jstor.org/ stable/985254

Simon, H. A. (2008). The sciences of the artificial (за ed.). The MIT Press.

Sosa, L. (2017) Diseño basado en sistemas complejos. El enfoque del diseño para transformar sociedades, sus ciudades y sus objetos. Universidad Autónoma de Nuevo León.

Thackara J. (2005). In the Bubble: Designing in a complex world. The MIT Press.

Weaver, W. (1948), "Science and complexity". En American Scientist (36). https://www.jstor.org/stable/27826254 